\title{
Proteindesign
}

\section{Photobodies: lichtaktivierbare Einzeldomänen-Antikörper}

BENEDIKT JEDLITZKE, ZAHIDE YILMAZ, HENNING D. MOOTZ

1INSTITUT FÜR BIOCHEMIE, UNIVERSITÄT MÜNSTER

\section{Photobodies are light-activatable single domain antibody fragments which show up to several-thousand fold impaired binding affinity in their photo-caged state. By irradiation with light, they regain their native antigen binding capacity. The endowed temporal and spatial control of photobodies over the binding event is expected to enable manifold potential applications in basic research, diagnostics and therapy.}

DOI: $10.1007 / \mathrm{s} 12268-020-1365-7$

(C) Die Autoren 2020

Antikörper sind aus der medizinisch angewandten Therapie und Diagnostik sowie aus den lebenswissenschaftlichen Forschungsdisziplinen mittlerweile nicht mehr wegzudenken. Ihre spezifische und hochaffine Bindung zu ihrem jeweiligen Antigen machen sie zu idealen Kandidaten für die zielgerichtete Detektion ihrer Antigene in komplexen Umgebungen wie z.B. im Blut oder Gewebe. Antikörper sind vergleichsweise große Proteine und bestehen aus zwei leichten (LC) und zwei schweren (HC) Ketten, die über Disulfidbrücken mit einander verknüpft sind (Abb. 1A). Diese Eigenschaften erschweren sowohl die rekombinante Produktion in Escherichia coli, als auch ihre definierte chemische Modifikation, z.B. die Konjugation mit Wirkstoffmolekülen oder
Farbstoffen. C. Hamers-Casterman et al. entdeckten im Immunsystem von Kamelen Antikörper, die nur aus schweren Ketten bestehen (Abb. 1B, [1]). Aus deren variabler Region wurden single-domain antibody-fragments (Nanobodies) entwickelt, also EinzeldomänenAntikörperfragmente, die überwiegend aus der für die Antigen-bindung notwendigen Paratop-Region bestehen (Abb. 1C). Diese sind im Vergleich zu den vollständigen Antikörpern etwa 10-mal kleiner und besitzen meist nur ein oder zwei Disulfidbrücken, die keine kettenverknüpfende Rolle einnehmen und für die strukturelle Stabilität oft entbehrlich sind. Aus diesen Gründen, ihrer leichten Produzierbarkeit in Bakterienzellen und ihrer guten Löslichkeit bieten sich Nanobodies für zahlreiche Einsatzgebiete an. Der-
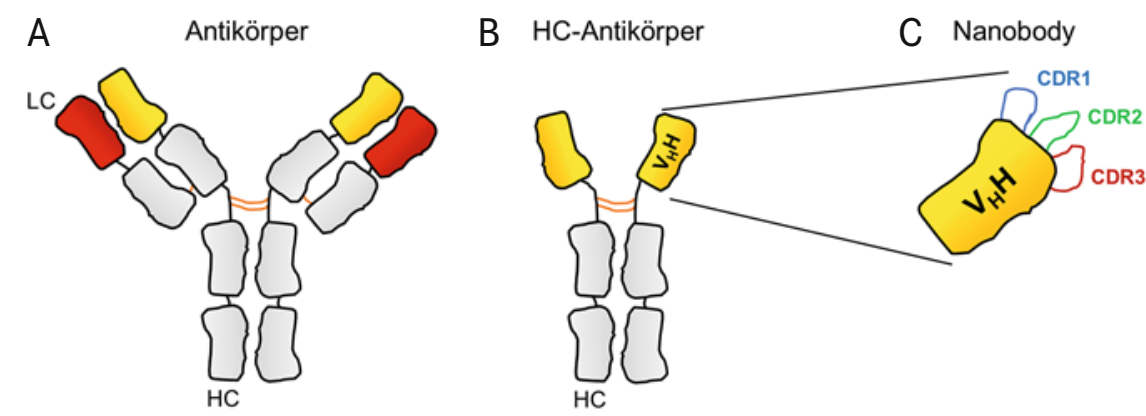

$\Delta$ Abb. 1: Schematische Darstellungen eines IgG-Antikörpers, (A) eines HC-Antikörpers und (B) eines Nanobodies (C). LC: light chain; HC: heavy chain.

zeit werden sie insbesondere in diagnostischen Bildgebungstechniken und als vielseitiges Werkzeug in der Grundlagenforschung eingesetzt.

Das Ziel unserer Arbeiten bestand darin, Nanobodies mithilfe von Licht zu aktivieren, sodass sie ihr Antigen erst nach Lichtbestrahlung binden können. Dies würde die lichtgesteuerte Kontrolle über die Antigenbindung erlauben und somit neue Anwendungsperspektiven eröffnen. Licht als Auslöser für Reaktionen oder Interaktionen von Biomolekülen $\mathrm{zu}$ verwenden ist in zweierlei Hinsicht eine elegante Lösung: Zum einen kann Licht bestimmter Wellenlängen ungehindert in Reaktionslösungen oder Gewebe eindringen, ohne physischen Schaden anzurichten. Zum anderen kann es örtlich und zeitlich gezielt eingesetzt werden. Eine vergleichbare Präzision ist mit anderen Aktivierungskonzepten nicht möglich.

\section{Tyrosine haben in Nanobodies eine besondere Bedeutung}

Unser Konzept zur Photoaktivierung von Nanobodies mit Licht besteht in dem Einbau einer photolabilen Schutzgruppe (photocage), um die Bindung an das Antigen durch den zusätzlichen sterischen Anspruch zu inhibieren [2]. Die Abspaltung der Schutzgruppe durch Lichtbestrahlung (de-caging) sollte die native Nanobody-Struktur wiederherstellen. Ein solcher Photo-caging-Ansatz ist für viele Moleküle bekannt. Im Falle von Proteinen handelt es sich dabei jedoch meist um Enzyme, die in der Regel effizient durch Modifizierung katalytisch wichtiger Seitenketten in der aktiven Tasche blockiert werden können. Für die Anwendung auf Nanobodies haben wir zunächst die Antigenbindungsdomänen (complementary determinding regions; CDRs) verschiedener Nanobodies genauer untersucht. Diese bestehen aus drei Schlaufen von stark variabler Aminosäuresequenz. Tyrosin ist darin eine der am häufigsten vertretenen Aminosäuren. Außerdem ist die Tyrosinseitenkette in den von uns untersuchten Kristallstrukturen oft in zentraler Position und direkt in Richtung der binden- 


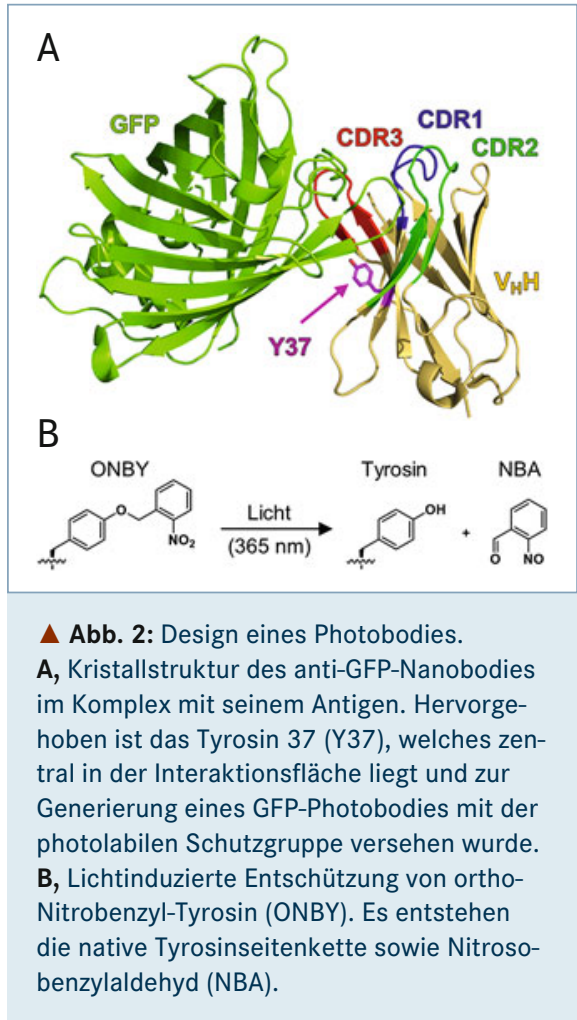

den Antigene gerichtet (Abb. 2A). Somit ist Tyrosin im besonderen Maß bei vielen Nanobodies an der spezifischen Antigen-Bindung beteiligt.

Auf der Suche nach einer photolabilen Schutzgruppe für Tyrosin wählten wir das Tyrosin-Derivat ortho-Nitrobenzyl-Tyrosin (ONBY). Die ONB-Gruppe kann bei Bestrahlung mit Licht der Wellenlänge $365 \mathrm{~nm}$ abgespalten werden, sodass Tyrosin als native Aminosäure zurückbleibt (Abb. 2B). Zudem ist ONBY eine der nicht-proteinogenen Aminosäuren (ncAA, non-canonical amino acid), die durch die Amber-Stoppcodon-Suppressionstechnologie als unnatürliche Aminosäure genetisch codiert, in rekombinante Proteine eingebaut werden kann [3].

\section{Einbau von photolabil geschütztem Tyrosin}

Durch den Einbau von ONBY anstelle eines Tyrosins in eine der drei CDR-Schleifen oder anderen zentralen Positionen der ParatopRegion konnten wir die Bindung von Nanobody und Antigen in bisher vier Beispielen um das bis zu 10.000fache verringern. Darunter sind Nanobodies gegen den in vielen Brustkrebsarten überexprimierten menschlichen epidermalen Wachstumsfaktor-Rezeptor 2 (HER2), sowie den epidermalen Wachstumsfaktor-Rezeptor (EGFR). Eine anschlieBende Bestrahlung mit Licht für weniger als
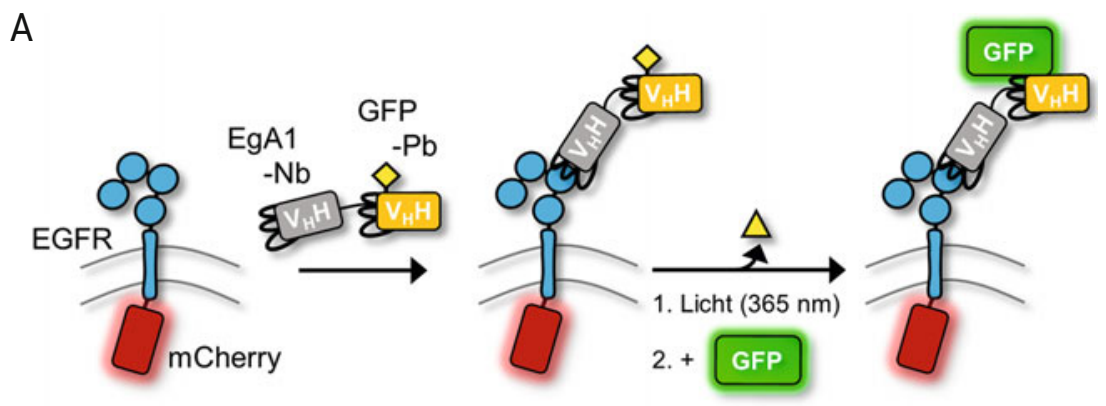

B
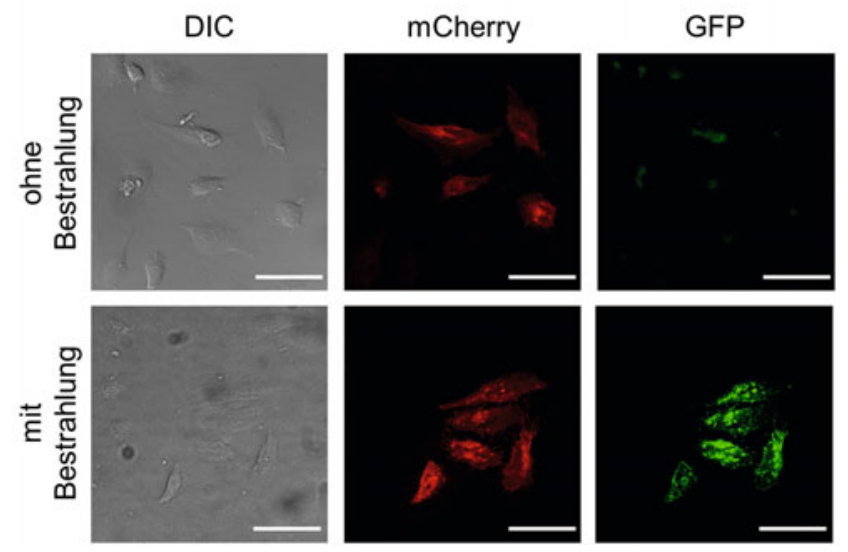

$\Delta$ Abb. 3: Lichtinduzierte Proteindimerisierung durch ein bispezifisches Nanobody-PhotobodyKonstrukt. A, Schematische Darstellung des Experiments, für das HeLa-Zellen transient mit einem EGFR-Fusionskonstrukt transfiziert wurden. In einem ersten Schritt bindet das bispezifische Nanobody-Photobody-Konstrukt über den EgA1-Nanobody an den auf der Zelloberfläche überexprimierten EGF-Rezeptor. Eine Bindung von GFP an den lichtgeschützten anti-GFP-Photobody (GFP-Pb) ist nicht möglich. Durch Lichtbestrahlung wird der anti-GFP-Photobody in einem zweiten Schritt entschützt und bindet anschließend GFP auf der Zelloberfläche. B, Analyse der lichtinduzierten Binding von GFP durch konfokale Fluoreszenzmikroskopie. Maßstab: $50 \mu \mathrm{m}$.

10 Sekunden brachte die ursprüngliche Interaktion zwischen Nanobody und Antigen zurück. Damit ist es erstmals gelungen, die spezifische Interaktion von Nanobody und Antigen durch den Austausch einer einzelnen Aminosäure im Nanobody gezielt zu unterdrücken und mit Licht zu aktivieren [2]. Solche lichtaktivierbaren Nanobodies nennen wir Photobodies. Die Gruppe von Amit Sachdeva entwickelte zeitgleich ein ähnliches Konzept [4].

\section{Bispezifische Photobodies als Mediatoren}

Um unseren Photobody-Ansatz in einem für Forschungskonzepte und potenziell therapeutisch relevanten Kontext zu demonstrieren, haben wir Nanobody-Photobody-Konstrukte entwickelt (Abb. 3A). Diese bestehen aus einem Nanobody-Teil der direkt an sein Antigen bindet, während der fusionierte Photobody-Teil erst mit Licht aktiviert werden muss. Letztendlich ließen sich damit zwei Proteine (die Antigene) lichtinduziert dimerisieren, was als Basis für viele Anwendungen denkbar ist. So könnte der Nanobody z.B. gegen einen Zelloberflächen-Rezeptor gerichtet sein, während der Photobody für ein zelltoxisches Antigen spezifisch ist. Da solche Oberflächenrezeptoren, z.B. in der Tumortherapie, oft in geringen Mengen auch auf gesunden Zellen exprimiert werden, wäre eine direkte Konjugation des Nanobodies mit dem zelltoxischen Antigen nicht wünschenswert. Ein gezieltes Ansteuern der gewünschten Zellen könnte jedoch erreicht werden, wenn das Nanobody-Photobody-Dimer zunächst an den Oberflächenrezeptor bindet und anschließend die gezielte Aktivierung des Photobodies durch Bestrahlung mit Licht lokal kontrolliert wird. Zelltoxisches Antigen würde nur an die bestrahlte Krebszelle binden ohne gesunde Zellen zu schädigen.

Zur Demonstration haben wir daher in einem Modellsystem HeLa-Zellen mit einem Plasmid für den in vielen Krebszellen überexprimierten EGF-Rezeptor transfiziert (Abb. 3B). Dazu haben wir ein bispezifisches 
Nanobody-Photobody-Fusionsprotein produziert, das zu einem Teil aus dem EGFR-bindenden EgA1-Nanobody [5] und im zweiten Teil aus einem anti-GFP-Photobody besteht. Der zugrundeliegende Nanobody ist gegen das grünfluoreszierende Protein (GFP) gerichtet [6] und wurde mittels ONBY-Einbau inaktviert [2]. Das Nanobody-PhotobodyFusionsprotein bindet nach Zugabe zu den transfizierten HeLa-Zellen an den EGFRezeptor (Abb. 3A). Nach Waschen der Zellen wurden diese mit Licht bestrahlt und GFP hinzugegeben. Tatsächlich haben unter diesen Bedingungen unserem DimerisierungsKonzept entsprechend nur die lichtbestrahlten HeLa-Zellen das GFP gebunden, während unbestrahlte Zellen oder solche ohne Nanobody-Photobody in der Fluoreszenz-Mikroskopie keine GFP-Anreicherung zeigten. Somit gelang es in Zellkulturexperimenten, eine Proteinanbindung mithilfe eines Nanobody-Photobody-Fusionsproteins, zeitlich und örtlich kontrolliert, mit Licht zu aktivieren [2]. Wir erwarten, dass sich mit diesem und ähnlichen Konzepten zahlreiche neue und spannende Anwendungen mit Photobodies realisieren lassen.

In Zukunft wird es erstrebenswert sein, Tyrosin-Derivate zu entwickeln, deren Entschützung bei längeren Wellenlängen möglich ist. So könnten mögliche zelltoxische Effekte vermindert und eine Penetration in tieferes Gewebe hinein ermöglicht werden, was den Anwendungsbereich von Photobodies nochmals erweitern würde (Mo 1073/7-1).

\section{Danksagung}

Die Arbeiten im Labor von Henning Mootz wurden durch die Deutsche Forschungsgemeinschaft (DFG) unterstützt (MO 1073/7-1).

\section{Literatur}

[1] Hamers-Casterman C, Atarhouch T, Muyldermans S et al. (1993) Naturally occurring antibodies devoid of light chains. Nature 363:446-448.

[2] Jedlitzke B, Yilmaz Z, Doerner W et al. (2020)

Photobodies: Light-Activatable Single-Domain Anti-body Fragments. Angew Chem Int Ed Engl 59:1506-1510.

[3] Deiters A, Groff D, Ryu Y et al. (2006) A Genetically Encoded Photocaged Tyrosine. Angew Chem Int Ed Engl 45:2728-2731.

[4] Bridge T, Shaikh SA, Thomas P et al. (2019) Site-Specific Encoding of Photoactivity in Antibodies Enables Light-

Mediated Antibody-Antigen Binding on Live Cells. Angew

Chem Int Ed Engl 58:17986-17993.

[5] Schmitz KR, Bagchi A, Roovers RC et al. (2013) Structural evaluation of EGFR inhibition mechanisms for nanobodies/ VHH domains. Structure 21:1214-1224.

[6] Kirchhofer A, Helma J, Schmidthals K et al. (2009)

Modulation of protein properties in living cells using nano bodies. Nat Struct Mol Biol 17:133-138.
Funding: Open Access funding provided by Projekt DEAL.

Open Access: Dieser Artikel wird unter der Creative Commons Namensnennung 4.0 International Lizenz veröffentlicht, welche die Nutzung, Vervielfältigung, erlaubt, sofern Sie den/die ursprünglichen Autor(en) und die Ouelle ordnungsgemäß nennen, einen Link zur Creative Commons Lizenz beifügen und angeben, ob Änderungen vorgenommen wurden. Die in diesem Artikel enthaltenen Bilder und sonstiges Drittmaterial unterliegen ebenfalls der genannten Creative Commons Lizenz, sofern sich aus der Abbildungslegende nichts anderes ergibt. Sofern das betreffende Material nicht unter der genannten Creative Commons Lizenz steht und die betreffende Handlung nich nach gesetzlichen Vorschriften erlaubt ist, ist für die oben aufgeführten Weiterverwendungen des Materials die Einwilligung des jeweiligen Lizenzinformation auf http://creativecommon

\section{Korrespondenzadresse}

Prof. Dr. Henning D. Mootz

Institut für Biochemie

Universität Münster

Wilhelm-Klemm-Str. 2

D-48149 Münster

Henning.Mootz@uni-muenster.de
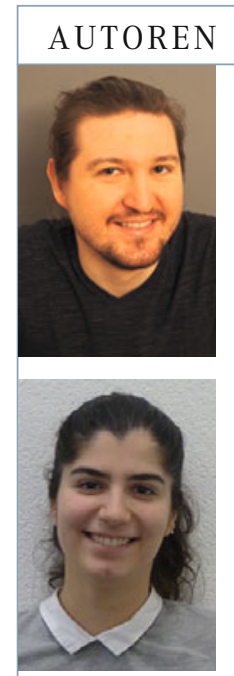

Zahide Yilmaz

2013-2018 Chemiestudium an der Universität Münster. 2017 Forschungsaufenthalt Universität Durham, UK. Seit 2018 Promotion am Institut für Biochemie der Universität Münster.

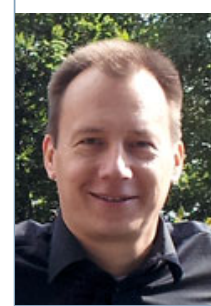

Henning D. Mootz

1990-1996 Chemiestudium in Wuppertal, Marburg und Canterbury, UK. 1996-1999 Promotion in Biochemie an der Universität Marburg mit Aufenthalt am Institut Pasteur, Paris. 1999-2003 Postdocs an der Universität Marburg und der Rockefeller University, New York, USA. 2003-2006 Emmy-Noether Stipendium an der Universität Marburg. 2006-2010 W2-Professor an der TU Dortmund. Seit 2010 W3-Professor an der Universität Münster.

\title{
Hier steht eine Anzeige.
}

\author{
Springer
}

